\title{
La alimentación ancestral amazónica y su impacto en el enfoque empresarial de la provincia de Morona Santiago
}

\section{The Amazon ancestral power and its impact on the approach empresarialde the province of Morona Santiago}

\author{
Morquecho Saltos, Julio*, Quezada Quezada, Patricio \\ Universidad Católica de Cuenca \\ Sede Macas, 140150, Ecuador \\ *jmorquechos@ucacue.edu.ec
}

\begin{abstract}
Resumen
La alimentación ancestral ha sido y es el origen de la salud plena. Su característica principal es el ser orgánica y desarrollada en una relación armoniosa entre la naturaleza y el hombre. En el presente trabajo se exponen las ideas fundamentales sobre dos especies amazónicas: el Mukindi o Rhynchohorus Palmarum y la Rolaquimba o Vasconcellea Monoica, las mismas que son parte fundamental de la dieta en las poblaciones amazónicas. El uso que han hecho los habitantes amazónicos de estas especies en relación a la alimentación y a la medicina, y la aceptación de las mismas entre la población, indican posibilidades de emprender en diversas formas de empresarialidad y la necesidad de continuar investigaciones más profundas sobre estas y otras especies amazónicas.
\end{abstract}

Palabras clave: Alimentación ancestral, Rhynchophorus Palmarum, Vasconcellea Monoica, enfoque empresarial.

\begin{abstract}
Ancestral feeding has been and still is the source of full health. Its main characteristic is that of being organic and grown in a harmonious relationship between nature and man. In the present work the fundamental ideas about two Amazonian species are presented: Mukinde or Rhynchophorus Palmarum and Rolaquimba or Vasconcellea Monoica, which are an essential part in the amazonian populations' diet. The use that the Amazonian inhabitants have made of these species in relation to food and medicine, and their acceptance among the population show possibilities of undertaking in various forms of entrepreneurship and the need to continue further research on these and other Amazonian species.s
\end{abstract}

Key words: ancestral feeding, Rhynchophorus Palmarum, Vasconcellea Monoica, business approach.

\section{Introducción}

\subsection{Antecedentes}

Desde tiempos muy remotos, las culturas ancestrales y últimamente la Shuar se ha sostenido alimentaria y medicinalmente con las especies nativas del Amazonas. Sin embargo, a nivel de ciencia se ha investigado poco en relación a la enorme biodiversidad de este lugar. (Paredes Loza, 2006)

Investigaciones sobre alimentación ancestral con la especie Rhynchophorus Palmarum y Vasconcellea Monoica hasta el momento no se han realizado, y en el marco de referencia consultado, se han encontrado pocos estudios sobre el uso alimentario de la primera especie, solo algunos estudios sobre su descripción. En el caso de la Rolaquimba tampoco existen estudios en absoluto, la especie investigada se encuentra categorizada como carica lo cual responde a estudios anteriores. Sin embargo, al momento se le ha categorizado como Vasconcellea Monoica, pero en realidad no cumple con las descripciones de la especie de Morona Santiago, cantón Morona. en la figura 1 se muestra la categorización de la especie tratada.

La categorización taxonómica se considera importante para referenciar las inclusiones o exclusiones de las especies en el contexto de un estudio investigativo.

\subsection{Naturaleza y alcance del problema investigado}

La situación socioeconómica de los pobladores de Morona Santiago es compleja, debido al bajo nivel de empleo y de emprendimientos empresariales, en consideración también conque trabajadores de todos los niveles y profesionales de otras provincias y países ocupan puestos laborales en las empresas e instituciones de la localidad. Además la visión empresarial y de emprendimiento no se ha desarrollado o no ha aportado de modo significativo en el contexto del desarrollo local, tanto desde el sector público como del privado ni de sus instituciones educativas en los 


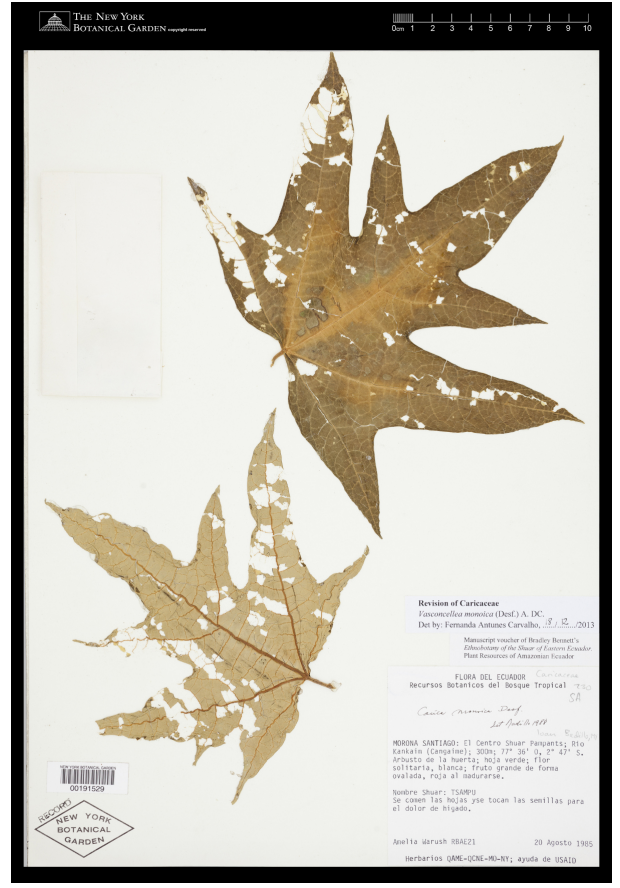

Figura 1. Vasconcellea monoica (Warush, 1985)

\section{diferentes niveles.}

La alimentación y la medicina ancestral aún no han sido reconocidas a plenitud, a pesar que en la Constitución del Ecuador elaborada en Montecristi en el año 2008, se reconocen los derechos de la madre naturaleza y el Buen Vivir o Sumak Kawsay. No obstante, los habitantes originarios de la Amazonía han perdido mucho de su sabiduría y conocimiento de las especies vegetales y animales, concomitante con el avance de la mal llamada civilización, sumado a la colonización y deforestación acelerada de los bosques primarios selváticos, al momento las especies de Rhynchoporus Palmarum y Vasconcellea Monoica son desconocidos, en algunos casos para los mismos pueblos originarios de la localidad.

\section{Revisión bibliográfica}

\subsection{La Alimentación ancestral}

La alimentación ancestral en el Ecuador es el fruto de las formas de producción propias de los Andes, que según las investigaciones en varios campos como la arqueología, la etnobiología, entre otras, han mostrado y demostrado que su producción alimentaria ha sido adaptativa y trasformacional en el contexto natural según los pisos climáticos y las necesidades de supervivencia en el entorno de las comunidades (Herrera, 1987)

Tanto la alimentación como la medicina ancestral nace de la relación profunda entre la Pachamama y el Runa (ser humano). La alimentación se sustenta en la producción orgánica y natural con la utilización de procesos de producción ancestrales para obtener alimentos sanos e integrales que satisfagan las necesidades biológicas, energéticas y de adaptación al entorno del ser humano.

En la Amazonía, la alimentación ancestral tiene el mismo fundamento de relación entre el ser humano y la naturaleza (Sachamama), con la diferencia que en algunos de los grupos humanos que moran los espacios selváticos profundos aún mantienen dicho equilibrio entre la producción y el consumo, teniendo presente siempre ese apego al equlibrio holístico entre el Runa y la Pachamama.

\subsubsection{El Rhynchophorus Palmarum}

La larva de Rhynchophorus Palmarum es conocida como Mukindi en la Amazonía ecuatoriana, en especial en la provincia de Morona Santiago. Este tipo de larva alimenticia ya sea cruda o preparada en su propia grasa (Vargas et al., 2013), es un alimento importante para varios grupos nativos de la Amazonía, los pueblos que la consumen indican que contiene grandes propiedades alimenticias y curativas, como se observa en la tabla 11.

Tabla 1

Parámetros en la composición nutricional de Rhynchophorus Palmarum.

\begin{tabular}{|l|l|}
\hline Parámetros & Resultados en porcentajes \\
\hline \hline Humedad & 59.60 \\
\hline Cenizas & 0.66 \\
\hline Proteínas & 9.49 \\
\hline Carbohidratos & 0.02 \\
\hline Calorías & $310.11 \mathrm{kcl} / 100 \mathrm{~g}$ \\
\hline Fibra bruta & 0.00 \\
\hline Calcio & $2.50 \mathrm{mg} / 100 \mathrm{~g}$ \\
\hline Magnesio & $25.00 \mathrm{mg} / 100 \mathrm{~g}$ \\
\hline Materia seca & 30.23 \\
\hline
\end{tabular}

El consumo de este tipo de invertebrados proporciona significativas cantidades de proteína animal, a los habitantes de las comunidades nativas, en especial los Shuar y Achuar durante los períodos más difíciles del año, como en la temporada de lluvias donde los peces y la caza son escasos. Según (Vargas et al., 2013) en el cantón Morona se consume esta especie, principalmente en los locales de comida típica amazónica y en eventos especiales como las diferentes ferias de la localidad. Su forma de consumo es en pinchos, ayampacos y de forma cruda (este tipo de consumo por lo general tiene una connotación medicinal).

En las observaciones realizadas en la provincia de Morona Santiago, las larvas de Mukindi se producen en las palmeras como la Mauritia Flexuosa (Giraldo, 1987), cuyo nombre común local es Acho. Para la producción de las larvas es necesario cortarla. Esta palmera para que inicie su etapa de aprovechamiento aproximadamente requiere de 10 a 15 años en lugares cultivados y en la selva profunda entre los 30 a 40 años, sin embargo la vida de esta especie puede llegar a más de 60 años de vida. 
En Venezuela se ha realizado un estudio sobre el Rhynchophorus Palmarum en el que se determinaron los tipos de nutrientes de esta especie y su relación con el consumo segun las edades de las personas.

Los resultados de dicho estudio permiten entender el contenido nutricional de una larva de Rhynchophorus Palmarum y su aplicación a infantes, niños y personas adultas, dentro de su dieta y requerimiento de nutrientes. A continuación la tabla 2 muestra las raciones humanas mínimas de nutrientes y contenido de nutrientes de la larva de Rhynchophorus Palmarum, estado Amazonas, Venezuela. (Según las categorias siguientes: A: Infantes entre 0,5 y 1 año; B: Niños entre 7 y 10 años; C: Hombres entre 25 a 50 años; D: Composición de una larva de R. Palmarum de 10 $\mathrm{g}$ de peso fresco. (Cerda et al., 1999)

Tabla 2

Nutrientes de Rhynchophorus Palmarum por cada larva de $10 \mathrm{~g}$ de peso fresco.

\begin{tabular}{|l|l|l|l|l|}
\hline Nutrientes & A & B & C & D \\
\hline \hline Proteina & 2,0 & 34 & 56 & 3,85 \\
\hline Calcio (g) & 540 & 800 & 800 & 0,10 \\
\hline Fosforo (mg) & 360 & 800 & 800 & 48 \\
\hline Magnesio (mg) & 70 & 350 & 350 & 31 \\
\hline Cobre (mg) & $0,5-07$ & $0,7-0,10$ & $2,0-3,0$ & 0,25 \\
\hline Zinc (mg) & 5 & 10 & 15 & 1,12 \\
\hline Hierro (mg) & 15 & 10 & 10 & 0,36 \\
\hline Manganeso (mg) & $0,5-0,7$ & $0,7-1,0$ & $2,5-5,0$ & 0,18 \\
\hline
\end{tabular}

Composición de nutrientes de Rhynchophorus Palmarum por cada larva de $10 \mathrm{~g}$ de peso fresco (Landívar Valverde, 2012)

\subsubsection{La Rolaquimba}

Entre muchas especies de usos alimenticios ancestrales se encuentra la rolaquimba. Como se indicó en la introducción, aun no existe una investigación específica sobre la especie localmente conocida, sin embargo se ha consultado sobre investigaciones que tratan el tema y se encontró su nombre científico como Vasconcellea Monoica (Desf.) A. DC (Encalada, 2003) expone en este informe que el fruto es comestible, aunque en la especie que se cultiva o se da de forma silvestre en Morona Santiago, sus frutos no son comestibles, se consideran tóxicos por la exploración empírica de las personas de la localidad.

De esta especie para la alimentación se utiliza solo las hojas de la rolaquimba. Uno de los platos típicos que se prepara entre los macabeos es el caldo de novios, el cual contiene esta especie como uno de sus componentes. Se utiliza para hacer ayampacos y también para hacer sopas y ensaladas con poca frecuencia. En estudios preliminares que el autor ha realizado se ha determinado niveles de hierro muy elevados(en trámite análisis nutricionales), a nivel de sanadores amazónicos se utiliza sus hojas para dietas como parte de la cosmovisión de la selva en la preparación de Uwishin (el sabio serpiente).

\subsection{Enfoque empresarial}

La complejidad de la sociedad industrial y posindustrial continúa siendo la generación de empleos. Para una gran mayoría de gobiernos en el mundo esta situación ha cambiado poco, en el caso de América Latina los gobiernos crearon puestos de trabajo innecesarios, con lo cual se creó la burocracia, como lo indica (Villegas and Varela, 2001)

Según indica (Paredes Loza, 2006) que "microempresa, es un negocio personal o familiar en el área de comercio, producción, o de servicios que tiene menos de 10 empleados (Choez, 2016), el cual es poseído y operado por una persona individual, una familia, o un grupo de personas de ingresos relativamente bajos, cuyo propietario ejerce un criterio independiente sobre productos, mercados y precios y además constituye una importante (si no la más importante) fuente de ingresos para el hogar".

\section{Métodos}

En este estudio se utilizó el método de análisis documental, a través del cual se valoraron las investigaciones trabajadas en relación al tema por los estudiantes como parte de la investigación formativa y del proceso de titulación en la carrera de Economía y Administración de Empresas respectivamente de la Universidad Católica de Cuenca, Sede Macas.

El estudio consideró un proyecto de investigación formativa del tercer año de Economía, con el título "Los usos medicinales del Rhynchophorus Palmarum" y un trabajo de grado de Administración de Empresas con el título "Proyecto de pre-factibilidad de la crianza del Mukindi (Rhynchophorus Palmarum), con visión empresarial para gastronomía típica y turística de la ciudad de Macas". Se revisaron de cada investigación: el problema, los objetivos, los resultados y su contribución al enfoque empresarial en la provincia de Morona Santiago, en Ecuador.

La investigación fue de tipo descriptiva, en la que se aplicaron cuestionarios, encuestas y guías de entrevistas para obtener la información sobre el tema relacionado con la oferta, demanda, precios y la aceptación del consumo del producto vivo y preparado de Rhynchophorus Palmarum. De igual manera se obtuvo información sobre la factibilidad del proceso productivo de las especies ancestrales medicinales.

\section{Resultados}

La investigación realizada en el año 2014, arrojó que el 85 por ciento de los encuestados están abiertos a la posibilidad de comercializar el Mukindi, Fig. 2.

Otro dato obtenido acerca del consumo del Mukindi o Rhynchophorus Palmarum es que ha sido utilizado por los pueblos nativos desde hace mucho tiempo dentro de su dieta, y en especial como medicina. 


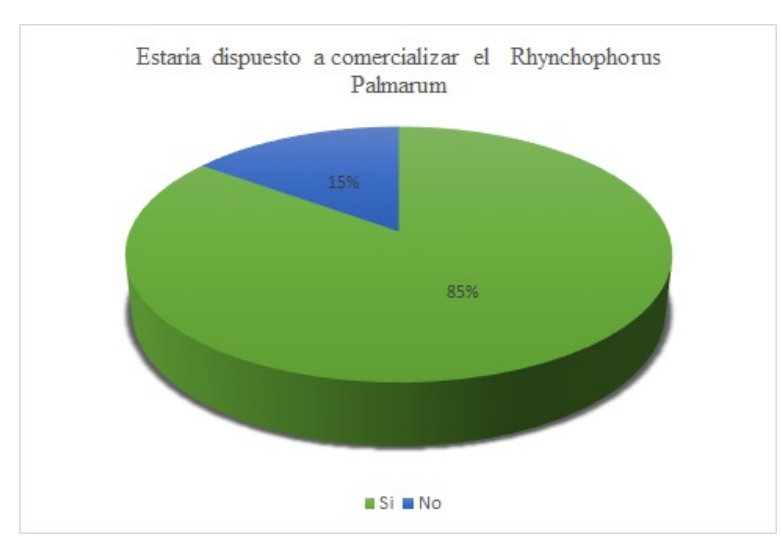

Figura 2. Posibilidad de comercialización

Al consultársele a los encuestados acerca de la introducción del Mukindi en su dieta alimenticia, 7 por ciento respondió que siempre, el 48 por ciento afirmó que a veces lo consumen, mientras que el 45 por ciento alegó qeu nunca lo hacen, aunqeu indicaron que era necesario dar a conocer la especie y sus bondades tanto desde el punto de vista nutritivo como medicinal. Fig. 3

Ha consumido usted mukinde (Rhynchophorus Palmarum)

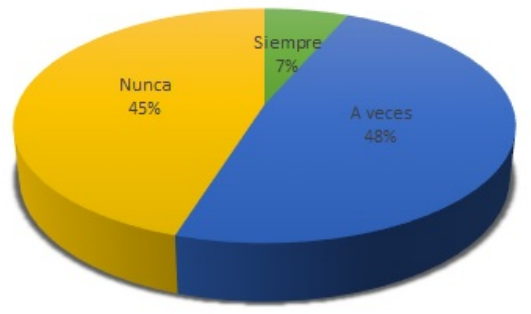

@Siempre $\|$ A veces $m$ Nunca

Figura 3. Estudio de mercado para el consumo de Mukindi (Rhynchophorus Palmarum)

En la dieta del pueblo mestizo se consideran importantes la Rolaquimba o Vasconcellea Monoica combinada con Mukindi, en especial en los ayampacos o maitos.

Del Mukindi se aprovecha en especial su grasa para preparar aceites y cremas de uso tópico con el fin de prevenir o disminuir los dolores reumáticos y artríticos, tambien el uso común que da el nativo Shuar es el consumo vivo del Mukindi, con el fin de enfrentar la anemia en los niños.

El estudio determinó los lugares más destacados en los que las personas han consumido el Mukindi: ver fig. 4 el 32 por cien consumio en restaurant de comidad típicas, el 20 por cien han consumido en centros turísticos que tienen servicio de alimentación tradicional, el 3 por cien lo ha hecho en lugares que ofrecen picaditas y el 45 por cien no ha consumido y posiblemente no lo conoce.

La Rolaquimba se utiliza más de la combinacion para

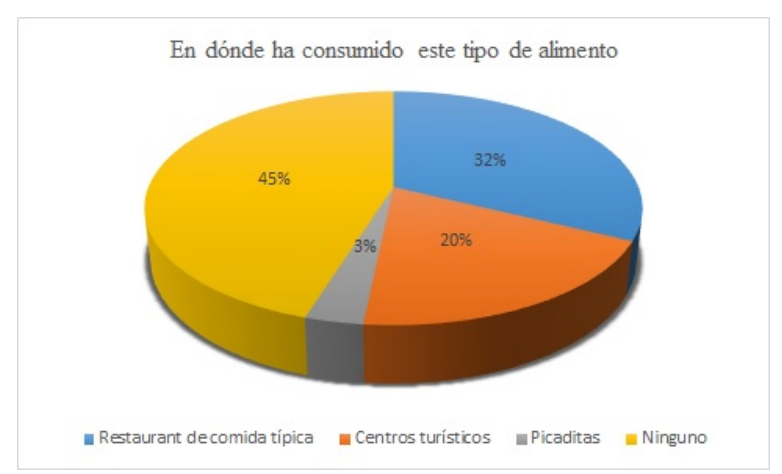

Figura 4. lugares en los que las personas encuestadas han consumido Mukindi(Rhynchophorus Palmarum)

los alimentos mencionados; sus hojas para corregir las deficiencias de hierro en los niños y adultos, la dosificación es establecida por los mayores y conocedores de la especie, con los frutos de la rolaquimba se elaboran compuestos orgánicos como pesticidas con el fin de prevenir la perdida de cultivos.

El uso de la medicina ancestral cobra vigencia constantemente, el diálogo intercultural y la inclusión paulatina de los sanadores ancestrales en las casas de salud, tanto hospitales como subcentros, permite entender que dicha medicina tiene su reconocimiento y vigencia en el contexto y desarrollo nacional como un país pluricultural. La medicina ancestral tiene como base la alimentación ancestral, de lo cual dan cuenta muchas investigaciones realizadas hasta la fecha, en los campos como la antropología, la etnobiología, y la misma medicina natural.

Las reservas de biodiversidad más completas están en la Amazonía, unido a ello se encuentran los pueblos custodios de los saberes tanto alimenticios como medicinales, a pesar del avance de la occidentalización, ellos se mantienen aún con algún conocimiento de su saber ancestral.

Las dos especies estudiadas en diferentes momentos, dan a entender que son aceptadas y consumidas por la población. Además muestran la posibilidad de generar emprendimientos en cuanto a la producción de las especie y la comercialización, en el caso del Mukindi se han hecho pruebas para su cría en cautiverio y a la Rolaquimba se la ha desarrollado en huertas familiares.

La cría en cautiverio del Mukindi se realizó en la parroquia Sevilla Don Bosco, las pruebas mostraron que para la alimentación óptima del desarrollo de la larva se utilizó papaya y caña de azúcar, con el 30 por ciento del peso de la larva. La incidencia de la muerte de las larvas en las pruebas se dio en un 50 por ciento del total inicial, la mayoría de muertes fue por canibalismo, por lo que se realizaron celdas individuales para alcanzar mejores resultados.

El cultivo de la Rolaquimba se realizó en huertos familiares y parcelas controladas por el autor principal de este artículo. Esta especie es silvestre y muy rústica, las pruebas se realizaron en varios tipos de terreno, y en 
todos ellos se desarrolla muy vigorosa, no presenta especies dañinas en su crecimiento ni madurez. La recoleccióbn de las hojas se realiza desde los tres meses de edad de la planta.
Recibido: 20 de Junio de 2016

Aceptado: 21 de abril de 2017

\section{Conclusiones}

Se debe aprovechar los recursos naturales de la Amazonía con base al conocimiento ancestral de la alimentación que admita fomentar una cultura de alimentación sana en base al desarrollo de procesos productivos en condiciones creadas por el hombre similares a las de la naturaleza, que permita generar una empresa que aporte al desarrollo de la provincia de Morona Santiago.

Se considera importante la investigación de la especie Vasconcellea Monoica con la finalidad de determinar los componententes nutricionales que permitan utilizarla de manera adecuada en la alimentación y la salud humana.

El aporte de los estudios realizados en relación a estas dos especies, permiten identificar posibles ideas de negocios que permitirian mejorar los ingreso de las personas que realicen emprendimientos de este tipo.

\section{Referencias}

Cerda, H., Martínez, R., Briceño, N., Pizzoferrato, L., Hermoso, D., and Paoletti, M. (1999). Cría, análisis nutricional y sensorial del picudo del cocotero rhynchophorus palmarum (coleoptera: Curculionidae), insecto de la dieta tradicional indígena amazónica. Ecotrópicos, 12(1):25-32.

Encalada, Claudio, I. P. U. d.(2003). Informe diversidad de frutales nativos comestibles Caricaceae-Solanaceae, fenologia, usos y recolección de germoplasmas en el sur del Ecuador.

Giraldo, L. E. U. (1987). Estudio preliminares de la fenología de la canangucha(mauritia flexuosa 1.f). Colombia amazonica, 2(2):57-81.

Herrera, N. (1987). La racionalidad campesina andina y la alimentación (el caso de la comuna de yana turro en la sierra central del ecuador). Agricultura y sociedad, (45):183-227.

Landívar Valverde, M. D. (2012). Evaluación del método de digestión alcalina para la extracción de grasa de larvas de rhynchophorus Palmarum $l$. $\mathrm{PhD}$ thesis.

Paredes Loza, M. C. (2006). Supervisión bancaria en el desarrollo futuro de las microfinanzas en el ecuador.

Choez, K. (1985). Los documentos comerciales y su aplicación en el comercio mercantil de las microempresas en la provincia de Santa Elena.

Vargas, G. E., Espinoza, G., Ruiz, C., and Rojas, R. (2013). Valor nutricional de la larva de rhynchophorus palmarum 1.: comida tradicional en la amazonía peruana. Revista de la Sociedad Química del Perú, 79(1):64-70.

Villegas, R. V. y Varela, R. (2001). Innovación empresarial: arte y ciencia en la creación de empresas. Pearson educación.

Warush, A. (1985). The new york botanical garden.
Morquecho, Julio: Docente a tiempo completo en la Universidad Católica de Cuenca Sede Macas, tecnólogo en Medicina Andina, Licenciado en Adminitración, Contador Público Autorizado, ingeniero empresarial, Magister en Gestión Empresarial

Patricio Quezada: Docente a tiempo completo en la Universidad Católica de Cuenca Sede Macas, Abogado, Doctor en Jurisprudencia, Especialista en Derecho Civil y comparado, Magister en Derecho Civil y Procesal Civil. equezadaq@ucacue.edu.ec 
